\title{
CONSTRUCTING APPROXIMATE FIBRATIONS
}

BY

\author{
T. A. CHAPMAN AND STEVE FERRY ${ }^{1}$
}

\begin{abstract}
In this paper two results concerning the construction of approximate fibrations are established. The first shows that there are approximate fibrations $p$ : $M \rightarrow S^{2}$ which are homotopic to bundle maps but which cannot be approximated by bundle maps. Here $M$ can be a compact $Q$-manifold or some topological $n$-manifold, $n \geqslant 5$. The second shows how to construct approximate fibrations $p: M \rightarrow B$ whose fibers do not have finite homotopy type, for any $B$ of Euler characteristic zero. Here $M$ can be a compact $Q$-manifold and $B$ only has to be an ANR, or $M$ can be an $n$-manifold, $n \geqslant 6$, and $B$ must then also be a topological manifold.
\end{abstract}

1. Introduction. Let $E$ and $B$ be locally compact separable metric ANRs and let $p$ : $E \rightarrow B$ be a proper map, i.e., the preimages of compacta are compact. We say that $p$ is an approproximate fibration provided that given any $X$ and maps $F: X \times[0,1] \rightarrow B$, $f: X \rightarrow E$ for which $F(x, 0)=p f(x)$, then for every $\varepsilon>0$ there exists a map $\tilde{F}$ : $X \times[0,1] \rightarrow E$ such that $\tilde{F}(x, 0)=f(x)$ and $p \tilde{F}(x, t)$ is $\varepsilon$-close to $F(x, t)$, for all $(x, t) \in X \times[0,1]$. This concept was introduced by Coram and Duvall in [10] as a generalization of the notion of a Hurewicz fibration. It has been studied extensively by several authors because approximate fibrations seem to arise more naturally than do Hurewicz fibrations or bundle maps when one studies certain topological manifold problems.

In this paper we will only be interested in approximate fibrations $p: E \rightarrow B$ for which $E=M^{k}, k \leqslant \infty$, where $M^{k}$ is a closed topological $k$-manifold for $k<\infty$, and is a compact Hilbert cube manifold (i.e., $Q$-manifold) for $k=\infty$. (Recall that a $Q$-manifold is a separable metric manifold modeled on the Hilbert cube $Q$.) Our main results are Theorems 1 and 2 where we show how to construct examples of approximate fibrations which are homotopic to bundle maps but which cannot be approximated by bundle maps, and also examples of approximate fibrations with nonfinite fibers.

In [17] Husch showed that an approximate fibration $p: M^{k} \rightarrow S^{1}$ can be approximated arbitrarily closely by a bundle map iff it is homotopic to a bundle map, $6 \leqslant k \leqslant \infty$. By a bundle map we mean a locally trivial bundle map with fiber an ANR. The following theorem shows that some restrictions are necessary in order to extend this result to more complicated bases. It also gives a negative answer to question QM15 of [15].

Received by the editors November 11, 1980 and, in revised form, March 29, 1982.

1980 Mathematics Subject Classification. Primary 57A15, 57A30, 57E25, 57A20.

$K e y$ words and phrases. Approximate fibration, $n$-manifold, $Q$-manifold.

'Both authors are supported by NSF Grant MCS-06929. The second author is an A. P. Sloan Fellow. 
THEOREM 1. For any $k, 5 \leqslant k \leqslant \infty$, there exists an approximate fibration $p$ : $M^{k} \rightarrow S^{2}$ which is homotopic to a bundle map but which cannot be approximated arbitrarily closely by bundle maps (or even Hurewicz fibrations).

REMARKS. (1) In the $Q$-manifold case the fiber of $p: M^{\infty} \rightarrow S^{2}$ can be taken to be any finite complex $F$ for which $K_{-1} Z \pi_{1}(F) \neq 0$, where $K_{-1}$ is the lower algebraic $K$-theoretic functor of Bass [2]. Calculations of Carter assure us that $K_{-1} Z \pi_{1}(F) \neq 0$, for $\pi_{1}(F) \cong Z_{2} \times Z_{7}[3]$.

(2) It is also true that if $K_{1-n} Z \pi_{1}(F) \neq 0$, then the proof of Theorem 1 gives us an approximate fibration $p: M^{\infty} \rightarrow S^{n}$ with fiber $F$ which is homotopic to a bundle map but which cannot be approximated arbitrarily closely by bundle maps.

(3) In the finite-dimensional case the fiber of $p: M^{k} \rightarrow S^{2}$ can be taken to be any closed manifold $F^{k-2}$ for which the duality involution, ${ }^{-}: K_{-1} Z \pi_{1}(F) \rightarrow K_{-1} Z \pi_{1}(F)$, which is induced by the involution $x \rightarrow x^{-1}$ on $\pi_{1}(F)$, does not act trivially or by multiplication by -1 . Fortunately for $n=2$ the example $\pi_{1}(F)=Z_{2} \times Z_{7}$ of Carter satisfies this property [3].

(4) Also for the finite-dimensional case if $F^{k-n}$ is chosen so that ${ }^{-}: K_{1-n} Z \pi_{1}(F)$ $\rightarrow K_{1-n} Z \pi_{1}(F)$ does not act trivially or by multiplication by -1 , then the proof of Theorem 1 gives us an approximate fibration $p: M^{k} \rightarrow S^{n}$ with fiber $F$ which is homotopic to a bundle map but which cannot be approximated arbitrarily closely by one.

(5) Certainly it would be nice to have techniques and calculations at our disposal which would extend Theorem 1 to include bases which are more complicated than spheres.

In order to state our next result we will have to recall some facts about approximate fibrations. If $p: E \rightarrow B$ is an approximate fibration of compact ANRs and $B$ is connected, then the homotopy fiber of $p$ is shape equivalent to all of the point-inverses of $p$. Also the homotopy fiber of $p$ is finitely dominated, i.e., is homotopy dominated by a finite complex. These facts follow easily from Proposition 2.3 of [6], or the reader can construct his own proofs. So it becomes a problem to determine which finitely dominated complexes can be realized as the homotopy fibers of approximate fibrations $p: M^{k} \rightarrow B$. In [12] Ferry showed that if $F$ is any finitely dominated complex, then there is an approximate fibration $p: M^{\infty} \rightarrow S^{1}$ with homotopy fiber $F$. In [14] Ferry constructed an example of an approximate fibration $p: M^{k} \rightarrow S^{1}, 6 \leqslant k<\infty$, whose homotopy fiber is nonfinite, i.e., does not have the homotopy type of a finite complex. The question was then raised of the possibility of obtaining approximate fibrations with nonfinite fibers over bases more complicated than $S^{1}[15$, QM13].

THEOREM 2. For any $k, 6 \leqslant k \leqslant \infty$, there exists an approximate fibration $p$ : $M^{k} \rightarrow B$ with nonfinite fiber, where

(1) $B$ is any compact connected ANR having Euler characteristic zero, if $k=\infty$,

(2) $B$ is a closed connected $n$-manifold, $k-n \geqslant 4$, having Euler characteristic zero, if $k<\infty$. 
REMARKS. (1) In the infinite-dimensional case the proof of Theorem 2 actually yields the following stronger result: If $B$ is a compact connected $A N R$ and $F$ is $a$ finitely dominated complex with Wall finiteness obstruction $\sigma(F) \in \tilde{K}_{0} Z \pi_{1}(F)$ which satisfies $\chi(B) \cdot \sigma(F)=0(\chi=$ Euler characteristic $)$, then $F$ is the homotopy fiber of some approximate fibration $p: M^{\infty} \rightarrow B$.

(2) In the finite-dimensional case the proof of Theorem 2 actually yields the following stronger result: If $B^{n}$ is a closed connected manifold and $F$ is a finitely dominated complex for which $\sigma(F)=\mu+(-1)^{k-n} \bar{\mu}$, for some $\mu \in \tilde{K}_{0} Z_{\pi_{1}}(F)$ and where ${ }^{-}: \tilde{K}_{0} Z \pi_{1}(F) \rightarrow \tilde{K}_{0} Z \pi_{1}(F)$ is the duality involution induced by the involution $x \rightarrow x^{-1}$ on $\pi_{1}(F)$, then there is an approximate fibration $p: M^{k} \rightarrow B^{n}$ whose homotopy fiber has finiteness obstruction equal to $\sigma(F)$ provided that $\chi(B) \cdot \sigma(F)=0$.

(3) A related problem is to determine just what finiteness obstructions are realizable in fibers of approximate fibrations of the above type. Specifically we ask: If $B$ is a compact connected $A N R$ and $G$ is a finitely presented group, what elements of $\tilde{K}_{0} Z G$ can be realized as the finiteness obstruction of the homotopy fiber $F$ of some approximate fibration $p: M^{\infty} \rightarrow B$, where $\pi_{1}(F)=G$ ? It is not hard to show that for any such $p: M^{\infty} \rightarrow B$ we must have $i_{*} \chi(B) \cdot \sigma(F)=0$, where $i$ is the inclusion $F \hookrightarrow M$ and $i_{*}: \tilde{K}_{0} Z \pi_{1}(F) \rightarrow \tilde{K}_{0} Z \pi_{1}(M)$ is induced by $\pi_{1}(F) \rightarrow \pi_{1}(M)$. Thus if $\pi_{1}(F) \rightarrow \pi_{1}(M)$ has a left inverse, then it follows that $\chi(B) \cdot \sigma(F)=0$ and so by Remark (1) above we have a complete determination of all possible $\sigma(F)$ 's in this case. Even if $\pi_{1}(F) \rightarrow \pi_{1}(M)$ does not have a left inverse then it is still possible to conjecture that $\chi(B) \cdot \sigma(F)$ must be zero. For example one can show that if $p$ : $M^{\infty} \rightarrow S^{2}$ is an approximate fibration with fiber $F$, then $2 \sigma(F)=0$. The general case seems difficult.

(4) As in Remark (3) above there is the realization problem for finite-dimensional manifolds. Again it seems difficult.

2. Preliminaries. All spaces in this paper will be locally compact, separable and metric. If $h_{t}: X \rightarrow Y$ is a homotopy, $0 \leqslant t \leqslant 1$, and $p: Y \rightarrow B$ is a map, then $h_{t}$ is said to be a $p^{-1}(\varepsilon)$-homotopy provided that each set $\left\{p h_{t}(x) \mid 0 \leqslant t \leqslant 1\right\}$ has diameter $<\varepsilon$. A proper map $f: X \rightarrow Y$ is said to be a $p^{-1}(\varepsilon)$-equivalence provided that there is a proper map $g: Y \rightarrow X$ so that $g f$ is proper $(p f)^{-1}(\varepsilon)$-homotopic to $\mathrm{id}_{X}$ and $f g$ is proper $p^{-1}(\varepsilon)$-homotopic to $\mathrm{id}_{Y}$. If $Y=B$ and $p=\mathrm{id}$, then $f$ is simply called an $\varepsilon$-equivalence.

For every pair of maps $f, g: X \rightarrow Y$ and $A \subset Y$ we say that $f=g$ over $A$ means that $f^{-1}(A)=g^{-1}(A)$ and the restrictions $f\left|f^{-1}(A), g\right| g^{-1}(A)$ are equal. For a given base $B$ and maps $f: X \rightarrow Y, p: X \rightarrow B$ and $q: Y \rightarrow B$, we say that $f$ is fiber preserving (f.p.) if $q f=p$.

If $f: X \rightarrow Y$ is a map of compacta, then we define the mapping cylinder of $f, M_{f}$, to be $(X \times[0,1])$ \& $Y / \sim$, where u means disjoint union and $\sim$ is the equivalence relation generated by $(x, 1) \sim f(x)$. We will represent $M_{f}$ as the union of $X \times[0,1)$ and $Y$, where $Y$ is the base and $X \equiv X \times\{0\}$ is the top. The collapse to the base is the retraction $c: M_{f} \rightarrow Y$ defined by $c \mid Y=\mathrm{id}$ and $c(x, t)=f(x)$.

Numerous concepts and results from $Q$-manifold theory will be used in the sequel such as $Z$-sets, $Z$-set unknotting, the Triangulation Theorem, and the Classification 
Theorem which relates the study of homeomorphisms on $Q$-manifolds to simple homotopy theory. We refer the reader to [8] for the appropriate $Q$-manifold theory.

A closed subset $A$ of a $Q$-manifold $M$ is said to be clean if $A$ is a $Q$-manifold and its topological boundary, $\operatorname{Bd}(A)$, is also a $Q$-manifold which is collared in $A$ and also in $M-\AA$ ( $\AA=$ topological interior of $A$ ). Similarly a closed subset $A$ of a PL manifold $M$ is said to be clean if $A$ is a PL submanifold which satisfies the above conditions and which lies in the interior of $M$.

We use $R^{n}$ to denote Euclidean $n$-space, with $R^{1}=R$, and we use $T^{n}=S^{1}$ $\times \cdots \times S^{1}$ to denote the $n$-torus. For any $r \geqslant 0$ we define $B_{r}^{n}=[-r, r]^{n}$.

Recall from [9] that to each homotopy equivalence $f: X \rightarrow Y$ of compact polyhedra (or compact ANRs [8]) we can assign a torsion $\tau(f) \in \mathrm{Wh}(Y)$ which vanishes iff $f$ is a simple homotopy equivalence. We will use several standard facts from simple homotopy theory such as the Sum Theorem, the formula for the torsion of a composition, etc. An excellent reference for all of this is [9].

We indicated in $\S 1$ that for each finitely dominated polyhedron $X$ we can assign an element $\sigma(X) \in \tilde{K}_{0} Z \pi_{1}(X)$ which vanishes iff $X$ is homotopy equivalent to a compact polyhedron. For simplicity we write $\tilde{K}_{0} Z \pi_{1}(X)=\tilde{K}_{0}(X)$ and by direct summing over path components we can extend the definitions to include nonconnected spaces. Thus $\tilde{K}_{0}$ (as well as Wh) becomes a homotopy functor from the category of spaces and maps to the category of abelian groups and homomorphisms. We will need the Sum Theorem for the Wall obstruction $\sigma(X)$. A good reference for this is [19, Chapter VI].

3. Proof of Theorem 1 (infinite-dimensional case). Before getting into the proof of Theorem 1 it will be convenient to establish the following lemma. Choose a point $x_{0} \in S^{2}$ and identify $R^{2}$ with $S^{2}-\left\{x_{0}\right\}$. In this section we will alternately use $\pi$ to denote projection to $T^{2}, R^{2}$ and $S^{2}$. The meaning should be clear from context.

LEMMA 3.1. For every $\varepsilon>0$ there exists $a \delta>0$ so that if $f: M \rightarrow R^{2} \times F$ is a $\pi^{-1}(\delta)$-equivalence, where $M$ is a $Q$-manifold and $F$ is a compact $Q$-manifold, then there exists a compact $Q$-manifold $N$ and a $\pi^{-1}(\varepsilon)$-equivalence $g: N \rightarrow S^{2} \times F$ for which $g=$ fover $B_{1}^{2} \times F$.

Proof. We will use mapping cylinder tricks that are similar to those used in [13]. Without loss of generality we may assume that $f$ is an inclusion and $M$ is a $Z$-set in $R^{2} \times F$. Also we may assume that for certain specific values of $t$ which arise in the following proof, $M \cap\left(B_{t}^{2} \times F\right)$ is clean in $M$. In particular it follows that the set $X=M \cup\left[\left(S^{2}-\dot{B}_{3}^{2}\right) \times F\right]$ is a compact $Q$-manifold because it is the union of the compact $Q$-manifolds $M \cap\left(B_{3}^{2} \times F\right)$ and $\left(S^{2}-\check{B}_{3}^{2}\right) \times F$ which meet in the $Q$ manifold $M \cap\left(\partial B_{3}^{2} \times F\right)$ which is a $Z$-set in each. Since $f$ is a $\pi^{-1}(\delta)$-equivalence it follows that $X \hookrightarrow S^{2} \times F$ is a $\pi^{-1}\left(\delta^{\prime}\right)$-domination rel $M$, i.e., there is a $\pi^{-1}\left(\delta^{\prime}\right)$-homotopy $h_{t}: S^{2} \times F \rightarrow S^{2} \times F$ for which $h_{0}=\mathrm{id}, h_{t} \mid M=\mathrm{id}$, and $h_{1}\left(S^{2} \times F\right) \subset X$. (The homotopy $h_{t}$ comes from [4] by using a controlled version of the homotopy extension theorem and the size of $\delta^{\prime}$ depends on the size of $\delta$.) Let $e=h_{1} \mid: X \rightarrow X$ and form the mapping telescope, $S_{e}$, which is the quotient space obtained from the disjoint union

$$
\cdots \text { ч }(X \times[-1,0]) \text { ч }(X \times[0,1]) \text { ч }(X \times[1,2]) \text { ч } \cdots
$$


by identifying $(x, n)$ in $X \times[n-1, n]$ with $(e(x), n)$ in $X \times[n, n+1]$. Note that $S_{e}$ is just the union of a countable number of copies of the mapping cylinder $M_{e}$. Here is a picture:

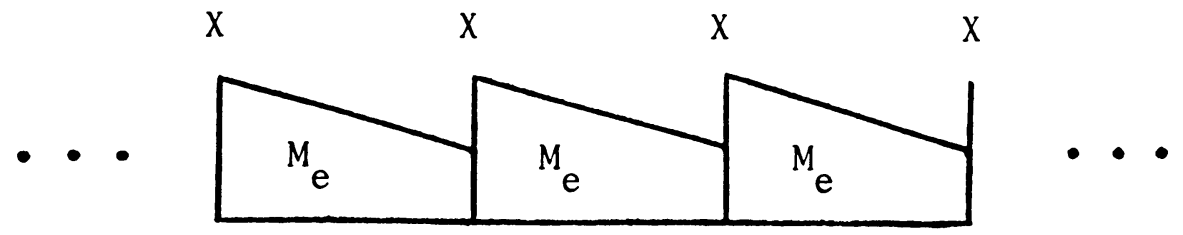

In a natural way $S_{e}$ may be set-wise identified with $X \times R$ (noncontinuously).

By the proof of Theorem 3.1 of [13] there is a homotopy equivalence $H: S_{e} \rightarrow$ $S^{2} \times F$ for which $H(x, 0)=x$, for $x \in X$, and $H(x, t)=x$, for all $(x, t)$ in $M \times R$. This is a (nonproper) $\pi^{-1}\left(\delta^{\prime \prime}\right)$-equivalence, where the size of $\delta^{\prime \prime}$ depends on the size of $\delta^{\prime}$. If $i: X \rightarrow S_{e}$ is defined by $i(x)=(x, 0)$, then we will construct $N$ and a nonproper $(\pi H)^{-1}\left(\varepsilon^{\prime}\right)$-equivalence $k: N \rightarrow S_{e}$ for which $k=i$ over $H^{-1}\left(B_{1}^{2} \times F\right)$, where the size of $\varepsilon^{\prime}$ depends on the size of $\delta^{\prime \prime}$. Once this is done we can define our desired $g$ : $N \rightarrow S^{2} \times F$ by $g=H K$.

Consider the decomposition $X=X_{1} \cup X_{2}$ where $X_{1}=X-\left(\stackrel{\circ}{2.5}^{2} \times F\right)$ and $X_{2}=$ $X \cap\left(B_{2.5}^{2} \times F\right)$. By our assumption that $M \cap\left(B_{2.5}^{2} \times F\right)$ is clean in $M$ we conclude that $X_{1}, X_{2}$ and $X_{0}=X_{1} \cap X_{2}$ are all $Q$-manifolds. For $\delta$ sufficiently small we have a decomposition $S_{e}=S_{e_{1}} \cup\left(X_{2} \times R\right)$, where $e_{1}=e \mid: X_{1} \rightarrow X_{1}$. The homotopy equivalence $H$ transfers the homotopy domination $X \hookrightarrow S^{2} \times F$ rel $M$ to give a homotopy domination $X \hookrightarrow S_{e}$ rel $M$. (We are identifying $X$ with $i(X)$ in $S_{e}$.) Using this it is easy to see that (for $\delta$ small) $X_{1} \hookrightarrow S_{e_{1}}$ is also a homotopy domination, thus the Wall obstruction $\sigma\left(S_{e_{1}}\right) \in \tilde{K}_{0}\left(S_{e_{1}}\right)$ is defined. We have

$$
\sigma\left(S_{e}\right)=\sigma\left(X_{0} \times R\right)=\sigma\left(X_{2} \times R\right)=0,
$$

so by the Sum Theorem for the Wall obstruction we conclude that $j_{*} \sigma\left(S_{e_{1}}\right)=0$, where $j_{*}: \tilde{K}_{0}\left(S_{e_{1}}\right) \rightarrow \tilde{K}_{0}\left(S_{e}\right)$ is induced by $j: S_{e_{1}} \hookrightarrow S_{e}$.

If $\delta$ is small enough the homotopy equivalence $H$, along with the functorality of $\tilde{K}_{0}$, provides us with a homomorphism

$$
\phi: \tilde{K}_{0}\left(S_{e}\right) \rightarrow \tilde{K}_{0}\left(S_{e_{1}} \cup\left(X_{2}-\left(\stackrel{\circ}{B}_{2.4}^{2} \times F\right)\right) \times R\right)
$$

so that $l_{*}=\phi j_{*}$, where $l$ is the inclusion $S_{e_{1}} \rightarrow S_{e_{1}} \cup\left(X_{2}-\left({\stackrel{\circ}{B_{2.4}}}^{2} \times F\right)\right) \times R$. From this we conclude that $l_{*} \sigma\left(S_{e_{1}}\right)=0$, and therefore

$$
\sigma\left(S_{e_{1}} \cup\left(X_{2}-\left(\stackrel{\circ}{2}_{2.4}^{2} \times F\right)\right) \times R\right)=0 .
$$

This means that the map $i: X \hookrightarrow S_{e}$ can be extended to our desired homotopy equivalence $k: N \rightarrow S_{e}$ by adding a compact polyhedron or, equivalently, a compact $Q$-manifold to $X-\left(\stackrel{\circ}{B}_{2.4}^{2} \times F\right)$ to make

$$
X-\left(\check{B}_{2.4}^{2} \times F\right) \hookrightarrow S_{e_{1}} \cup\left(X_{2}-\left(\stackrel{\circ}{2}_{2.4}^{2} \times F\right)\right) \times R
$$

into a homotopy equivalence. It is easy to get $k$ to be a $(\pi H)^{-1}\left(\varepsilon^{\prime}\right)$-equivalence simply by choosing the identification $R^{2} \equiv S^{2}-\left\{x_{0}\right\}$ so that the diameter of $S^{2}-\dot{B}_{2}^{2}$ is small. 
Proof of Theorem 1 (Infinite-dimensional CASE). We start by choosing any compact connected $Q$-manifold $F$ for which $K_{-1} Z \pi_{1}(F) \neq 0$. Since $K_{-1} Z \pi_{1}(F)$ is a direct summand of $\mathrm{Wh}\left(T^{2} \times F\right)=\mathrm{Wh} Z \pi_{1}\left(T^{2} \times F\right)$ [1] we can choose a compact $Q$-manifold $M_{1}$ and a homotopy equivalence $f_{1}: M_{1} \rightarrow T^{2} \times F$ whose Whitehead torsion, $\tau\left(f_{1}\right)$, is a nonzero element of the subgroup $K_{-1} Z \pi_{1}(F)$ of $\mathrm{Wh}\left(T^{2} \times F\right)$. Such torsions are invariant under passage to standard finite covers, so we may assume that $f_{1}$ is a $\pi^{-1}\left(\varepsilon_{1}\right)$-equivalence, where $\pi=$ proj: $T^{2} \times F \rightarrow T^{2}$ and $\varepsilon_{1}$ is a small number (compare with Lemma 7.3 of [6]).

Let $e: R \rightarrow T^{1}$ be the covering map defined by $e(x)=e^{\pi i x}$, thus giving the covering map $e^{n}=e \times \cdots \times e: R^{n} \rightarrow T^{n}$. Form the pull-back,

$$
\begin{array}{ccc}
M_{2} & \stackrel{f_{2}}{\rightarrow} & R^{2} \times F \\
\downarrow & & \downarrow e^{2} \times \text { id } \\
M_{1} & \stackrel{f_{1}}{\rightarrow} & T^{2} \times F,
\end{array}
$$

where $M_{2} \subset M_{1} \times\left(R^{2} \times F\right)$ consists of those elements $(x, y)$ of $M_{1} \times\left(R^{2} \times F\right)$ for which $f_{1}(x)=\left(e^{2} \times\right.$ id $)(y)$. The map $f_{2}$ is a projection map and it is a $\pi^{-1}\left(\varepsilon_{2}\right)$-equivalence, where $\varepsilon_{2}$ depends on $\varepsilon_{1}$. Also the map $M_{2} \rightarrow M_{1}$ is an immersion, and therefore $M_{2}$ is a $Q$-manifold. These are standard facts about the pull-back construction which have appeared several times in the literature (see, for example, the proof of Theorem 2 of [6]). Using Lemma 3.1 we can find a $\pi^{-1}\left(\varepsilon_{3}\right)$-equivalence $f_{3}: M_{3} \rightarrow S^{2} \times F$ where $M_{3}$ is a compact $Q$-manifold, $f_{3}=f_{2}$ over $B_{1}^{2} \times F$, and $\varepsilon_{3}$ depends on $\varepsilon_{2}$. For $k$ large and $\varepsilon_{3}$ small we can choose a clean manifold $C \subset M_{3}$ which lies in $f_{3}^{-1}\left(\left(S^{2}-\dot{B}_{k}^{2}\right) \times F\right)$ for which the inclusion-induced homomorphism $\mathrm{Wh}(C) \rightarrow \mathrm{Wh}\left(M_{3}\right)$ is surjective.

We can find a compact $Q$-manifold $\tilde{C}$ containing $C$ as a $Z$-set so that there exists a strong deformation retraction $r: \tilde{C} \rightarrow C$ whose torsion, $\tau(r) \in \mathrm{Wh}(C)$, is sent to $-\tau\left(f_{3}\right) \in \mathrm{Wh}\left(S^{2} \times F\right)$ under the composition

$$
\mathrm{Wh}(C) \rightarrow \mathrm{Wh}\left(M_{3}\right) \stackrel{\left(f_{3}\right)^{*}}{\rightarrow} \mathrm{Wh}\left(S^{2} \times F\right) .
$$

Let $M=M_{3} \cup \tilde{C}$ (sewn together along $C$ ), which is still a $Q$-manifold, and let $f$ : $M \rightarrow S^{2} \times F$ be the homotopy equivalence obtained by first retracting $\tilde{C}$ to $C$ via $r$, and then applying $f_{3}$. Observe that $f$ is a $\pi^{-1}(\varepsilon)$-equivalence, where $\varepsilon$ is small if $k$ is large and $\varepsilon_{3}$ is small. Also we have $\tau(f)=0$ by the formula for the torsion of a composition [9, p. 72], so $f$ is homotopic to a homeomorphism $h: M \rightarrow S^{2} \times F$ by the Classification Theorem of [8]. In the language of [6] the map $\pi f: M \rightarrow S^{2}$ is $\varepsilon^{\prime}$-fibration, where $\varepsilon^{\prime}$ is small if $\varepsilon$ is small. Thus by Theorem 1 of [6] we can choose $\varepsilon^{\prime}$ small enough so that $\pi f$ is close to an approximate fibration $p: M \rightarrow S^{2}$. We have a homotopy $p \simeq \pi f \simeq \pi h$, the latter map being a bundle map. So we will be done when we have shown that $p$ is not close to a bundle map.

That $p$ is not close to a bundle map is a consequence of the proof of Theorem 2 of [6]. We outline the proof. Embed $S^{1} \times R^{1}$ in $S^{2}$. Restricting $p$ to the inverse image of $S^{1} \times R^{1}$ gives an approximate fibration over $S^{1} \times R^{1}$. Controlled engulfing and 
Theorem 1 of [6] produce an approximate fibration over $T^{2}$ and a controlled homotopy equivalence from the total space of this approximate fibration to $T^{2} \times F$. The torsion of this homotopy equivalence lies in $\mathrm{Wh}\left(T^{2} \times F\right)$. A major portion of the proof of Theorem 2 of [6] consists of showing that the $\tilde{K}_{-1}$ component of the torsion is independent of the original embedding and of the wrapping up process.

It is now clear that $p$ is not approximated by a bundle map. If this were possible, then one could easily perform the embedding and wrapping up process so that the $\tilde{K}_{-1}$ component of the resulting torsion would be zero. On the other hand, the process can clearly be performed in such a way as to recover the original manifold $M_{1}$ and homotopy equivalence $f_{1}$, so the $\tilde{K}_{-1}$ component of the torsion is nonzero.

4. Proof of Theorem 1 (finite-dimensional case). In this section we will be dealing with PL $h$-cobordisms $(W, N)$ and it will always be understood that $\partial W=\partial_{0} W \cup N$, where $\partial_{0} W \cap N=\varnothing$. Also we will always have a given proper map $\pi$ of $N$ to some base space $B$, and by a $\pi^{-1}(\varepsilon)$-sdr (sdr $\equiv$ strong deformation retraction) we will mean a proper retraction $f: W \rightarrow N$ which is $(\pi f)^{-1}(\varepsilon)$-homotopic to id ${ }_{W}$ rel $N$. As in our treatment of the infinite-dimensional case in $\$ 3$ it will be convenient to first establish an extension lemma. Our basic notation is the same as in $\S 3: \pi$ denotes projection to $T^{2}, R^{2}$ or $S^{2}$, and we assume the identification $R^{2} \equiv S^{2}-\left\{x_{0}\right\}$.

LEMMA 4.1. For every $\varepsilon>0$ there exists a $\delta>0$ so that if $\left(W^{k+1}, R^{2} \times F\right)$ is an $h$-cobordism $(k \geqslant 5), f: W \rightarrow R^{2} \times F$ is a $\pi^{-1}(\delta)-s d r$, and $\partial_{0} W \hookrightarrow W$ is $a(\pi f)^{-1}(\delta)-$ equivalence, then there exists an $h$-cobordism $\left(V, S^{2} \times F\right)$ and $a \pi^{-1}(\varepsilon)$-sdr $g: V \rightarrow S^{2}$ $\times F$ for which $\partial_{0} V \hookrightarrow V$ is $a(\pi g)^{-1}(\varepsilon)$-equivalence and $g=$ fover $B_{1}^{2} \times F$.

Proof. Using the geometrical connectivity result of $[18$, p. 308] we may assume that $W$ is a handlebody structure on $R^{2} \times F$ consisting of two layers of handles of indices $k-2$ and $k-1$, where the image of each handle under the map $\pi f: W \rightarrow R^{2}$ has a small diameter. Let $W^{\prime}$ be the union of $S^{2} \times F$ and all handles of $W$ which meet $(\pi f)^{-1}\left(B_{3}^{2}\right)$. Then $W^{\prime}$ is a handlebody structure on $S^{2} \times F$ and the restriction $f \mid: W^{\prime} \rightarrow S^{2} \times F$ is a $\pi^{-1}(\delta)$-equivalence over $B_{2}^{2} \times F$. It then follows from the trick on p. 324 of [18] that we can alter this handlebody structure over $\left(S^{2}-\dot{B}_{2}^{2}\right) \times F$ to form a new $h$-corbordism $\left(V, S^{2} \times F\right)$, and if $S^{2}-\check{B}_{2}^{2}$ has a sufficiently small diameter there is a $\pi^{-1}(\varepsilon)$-sdr $g: V \rightarrow S^{2} \times F$ which fulfills our requirements.

Proof of Theorem 1 (Finite-dimensional CASE). We follow fairly closely the proof of the infinite-dimensional case of $\S 3$. Choose a compact connected manifold $F^{k-2}$ for which the duality involution ${ }^{-}: K_{-1} Z \pi_{1}(F) \rightarrow K_{-1} Z \pi_{1}(F)$ does not act trivially or by multiplication by -1 . As indicated in $\$ 1$ all we need to do is choose $\pi_{1}(F)=Z_{2} \times Z_{7}$ to fulfill these requirements. Thus we can choose an element $\tau \in K_{-1} Z \pi_{1}(F) \subset \mathrm{Wh}\left(T^{2} \times F\right)$ for which

$$
\bar{\tau} \neq\left\{\begin{array}{cc}
\tau & \text { if } k \text { is even, } \\
-\tau & \text { if } k \text { is odd. }
\end{array}\right.
$$

Now form an $h$-cobordism $\left(W_{1}, T^{2} \times F\right)$ such that there exists a $\operatorname{sdr} f_{1}: W_{1} \rightarrow T^{2} \times F$ which has torsion $\tau$. This of course means that $\partial_{0} W_{1} \leftrightarrow W_{1}$ is also a homotopy equivalence. Here $\partial_{0} W_{1}$ denotes the other boundary component of $W_{1}$. If $u: T^{2} \rightarrow T^{2}$ 
is any standard finite cover we can form the pull-back

$$
\begin{array}{ccc}
W_{2} & \stackrel{f_{2}}{\rightarrow} & T^{2} \times F \\
\downarrow & & \downarrow u \times \mathrm{id} \\
W_{1} & \stackrel{f_{1}}{\rightarrow} & T^{2} \times F .
\end{array}
$$

If $u$ is a large enough cover we conclude that $f_{2}$ is a $\pi^{-1}\left(\varepsilon_{2}\right)-\operatorname{sdr}$, where $\varepsilon_{2}$ is a small number. By the same token it follows that we can also choose $u$ large enough so that $\partial_{0} W_{2} \hookrightarrow W_{2}$ is a $\left(\pi f_{2}\right)^{-1}\left(\varepsilon_{2}\right)$-equivalence.

The next step is to form the pull-back,

$$
\begin{array}{ccc}
W_{3} & \stackrel{f_{3}}{\rightarrow} & R^{2} \times F \\
\downarrow & & \downarrow e^{2} \times \mathrm{id} \\
W_{2} & \stackrel{f_{2}}{\rightarrow} & T^{2} \times F .
\end{array}
$$

Then $\left(W_{3}, R^{2} \times F\right)$ is an $h$-cobordism, $f_{3}$ is a $\pi^{-1}\left(\varepsilon_{3}\right)$-sdr, and $\partial_{0} W_{3} \hookrightarrow W_{3}$ is a $\left(\pi f_{3}\right)^{-1}\left(\varepsilon_{3}\right)$-equivalence, where $\varepsilon_{3}$ depends on $\varepsilon_{2}$. Using Lemma 4.1 we can find an $h$-cobordism $\left(W_{4}, S^{2} \times F\right)$ and a $\pi^{-1}\left(\varepsilon_{4}\right)$-sdr $f_{4}: W_{4} \rightarrow S^{2} \times F$ for which $\partial_{0} W_{4} \hookrightarrow W_{4}$ is a $\left(\pi f_{4}\right)^{-1}\left(\varepsilon_{4}\right)$-equivalence and $f_{4}=f_{3}$ over $B_{1}^{2} \times F$, where $\varepsilon_{4}$ depends on $\varepsilon_{3}$. If $r$ is large and $\varepsilon_{4}$ is sufficiently small we can find a clean PL submanifold $C^{k}$ of $\partial_{0} W_{4}$ which lies in $f_{4}^{-1}\left(\left(S^{2}-\dot{B}_{r}^{2}\right) \times F\right)$ and for which the inclusion-induced homomorphism $\mathrm{Wh}(C) \rightarrow \mathrm{Wh}\left(W_{4}\right)$ is surjective. Let $(\tilde{C}, C)$ be an $h$-cobordism rel $\partial C$ for which the torsion of the sdr $s: \tilde{C} \rightarrow C$ is sent to $-\tau\left(f_{4}\right)$ under the composition

$$
\mathrm{Wh}(C) \rightarrow \mathrm{Wh}\left(W_{4}\right) \stackrel{\left(f_{4}\right)}{\rightarrow}{ }^{*} \mathrm{Wh}\left(S^{2} \times F\right) .
$$

Let $W=W_{4} \cup \tilde{C}$ (sewn together along $C$ ), which defines a new $h$-cobordism $\left(W, S^{2} \times F\right)$. The sdr $f: W \rightarrow S^{2} \times F$, which is defined by first retracting $\tilde{C}$ to $C$ and then applying $f_{4}$, is a $\pi^{-1}(\varepsilon)$-sdr for $\varepsilon$ small provided that $r$ is large and $\varepsilon_{4}$ is small. Also we have $\tau(f)=0$, so by the $s$-cobordism theorem the cobordism $\left(W, S^{2} \times F\right)$ is trivial, i.e., $\left(W, S^{2} \times F\right)$ is homeomorphic to $\left(S^{2} \times F \times[0,1]\right.$, $\left.S^{2} \times F\right)$.

Let $M=\partial_{0} W$ and note that, in the language of [7], the composition $M \stackrel{i}{\rightarrow} W \stackrel{\pi f}{\rightarrow} S^{2}$ is an $\varepsilon^{\prime}$-fibration, where $\varepsilon^{\prime}$ is small if $\varepsilon$ is small. By Theorem 1 of [7] we conclude that $\pi f i: M \rightarrow S^{2}$ is close to an approximate fibration $p: M \rightarrow S^{2}$. Since the cobordism $\left(W, S^{2} \times F\right)$ is trivial we conclude that $p$ is homotopic to a bundle map. We will be done when we have shown that $p$ is not close to a bundle map. To do this we can multiply everything by $Q$ to obtain $Q$-manifolds, and then repeat the proof of the corresponding $Q$-manifold assertation of $\$ 3$. To carry this out we need to know that the torsion of the composition $\partial_{0} W_{1} \stackrel{j}{\rightarrow} W_{1} \rightarrow f_{1} \rightarrow T^{2} \times F$ is nonzero. Recall from [16, p. 273] that we have the formula $\tau(j)=(-1)^{k} \overline{t(l)}$, where $l: T^{2} \times F \hookrightarrow W_{1}$ and ${ }^{-}$: $\mathrm{Wh}\left(W_{1}\right) \rightarrow \mathrm{Wh}\left(W_{1}\right)$ is the duality involution that is compatible with the one on 
$K_{-1} Z \pi_{1}(F)$ that has already been mentioned. From this it is easy to calculate $\tau\left(f_{1} j\right)=\tau+(-1)^{k+1} \bar{\tau}$, which is nonzero by our choice of $\tau$.

5. Controlled finiteness theorems. The purpose of this section is to establish two results (Theorems 5.2 and 5.3) which will be needed in the proof of Theorem 2. Specifically we will examine the following question: If $B$ is a compact connected polyhedron and $F$ is a finitely dominated polyhedron, when is there a compact polyhedron $K$ and $a p^{-1}(\varepsilon)$-equivalence $K \rightarrow B \times F$, where $p=\operatorname{proj}: B \times F \rightarrow B$ and $\varepsilon$ is small? In Theorem 5.2 we show that this is true iff $\chi(B) \cdot \sigma(F)=0$, where $\sigma(F) \in \tilde{K}_{0}(F)$ is the Wall obstruction of $F$ and $\chi(B)$ is the Euler characteristic of $B$. Theorem 5.3 is merely a refinement of Theorem 5.2 with dimension estimates.

Before we establish these results we will first have to prove a lemma. For notation let $B$ be a compact connected polyhedron and let $p: E \rightarrow B$ be a PL map which is an approximate fibration, where $E$ is also a compact polyhedron. For a fixed basepoint $* \in B$ we assume that there is a retraction $r: E \rightarrow p^{-1}(*)$. Now let $p_{1}$ : $E_{1} \rightarrow B$ be another such PLapproximate fibration so that $E_{1}$ contains $E$ as a subpolyhedron and so that there is a f.p. retraction $f_{1}: E_{1} \rightarrow E$ which is f.p. homotopic to id rel $E$. This can be rephrased by saying that $f_{1}$ is a f.p. sdr. For each simplex $\Delta \subset B$ (in a fixed triangulation of $B$ ) the homotopy equivalence $f_{1} \mid$ : $p_{1}^{-1}(\Delta) \rightarrow p^{-1}(\Delta)$ gives us a torsion $\tau\left(f_{1} \mid\right) \in \mathrm{Wh}\left(p^{-1}(\Delta)\right)$ and we let $\tau_{\Delta}$ be the image of $\tau\left(f_{1} \mid\right)$ in $\mathrm{Wh}\left(p^{-1}(*)\right)$ under the composition

$$
\mathrm{Wh}\left(p^{-1}(\Delta)\right) \rightarrow \mathrm{Wh}(E) \stackrel{r *}{\rightarrow} \mathrm{Wh}\left(p^{-1}(*)\right) .^{2}
$$

If $B_{1}$ is any subcomplex of $B$, then we inductively define $\tau_{B_{1}} \in \mathrm{Wh}\left(p^{-1}(*)\right)$ by $\tau_{B_{1}}=\tau_{B_{2}}+\tau_{\Delta}-\tau_{\partial \Delta}$, where $B_{1}=B_{2} \cup \Delta$ and $\Delta \cap B_{2}=\partial \Delta$. It is easy to show that if $\tau\left(f_{1}\right) \in \mathrm{Wh}(E)$ is the usual torsion of $f_{1}$, then $\tau\left(f_{1}\right)$ is the image of $\tau_{B}$ under the composition $\mathrm{Wh}\left(p^{-1}(*)\right) \rightarrow \mathrm{Wh}(E)$.

LEMMA 5.1. If $\tau_{B}=0$, then for every $\varepsilon>0$ there exists a homeomorphism $h$ : $E_{1} \times Q \rightarrow E \times Q$ which is $(p \circ \text { proj })^{-1}(\varepsilon)$-homotopic to $f_{1} \times \mathrm{id}_{Q}$.

PROOF. There is a trick of trading adjacent cancelling torsions which will be useful. To explain this we look at the simplified case $B=[0,1]$ (a single 1-simplex) and show how torsions can be traded to concentrate $\tau_{B}$ over $*$, which we take to be $1 \in[0,1]$. In what follows all torsions are in $\mathrm{Wh}\left(p^{-1}(*)\right)$. Choose a fine subdivision of [0, 1], $0=t_{0}<t_{1}<\cdots<t_{2 k+1}=1$, and form $E_{2}=E_{1} \cup\left(\cup_{i=0}^{2 k+1} A_{i}\right)$, where the $A_{i}$ are disjoint compact polyhedra so that $A_{i}$ meets $E_{1}$ in the subpolyhedron $p^{-1}\left(t_{i}\right)$. Also we want there to be a PL sdr $r_{i}: A_{i} \rightarrow p^{-1}\left(t_{i}\right)$ so that

(1) $\tau\left(r_{2 i}\right)+\tau\left(f_{1} \mid p_{1}^{-1}\left(t_{2 i}\right)\right)=0,0 \leqslant i \leqslant k$,

(2) $\tau\left(r_{2 i+1}\right)+\tau\left(r_{2 i}\right)=0,0 \leqslant i \leqslant k$.

The sdr's $r_{i}$ piece together to give us a PL sdr $f_{2}: E_{2} \rightarrow E_{1}$ so that $p_{2}=p_{1} f_{2}: E_{2} \rightarrow B$ is a $\mathrm{PL}$ approximate fibration. By (2) we have formed $E_{2}$ by adding torsions to $E_{1}$ in adjacent cancelling pairs. It follows from the Classification Theorem of [8], along

\footnotetext{
${ }^{2}$ The reader should note that this is an isomorphism.
} 
with Z-set unknotting, that $f_{2} \times$ id $Q: E_{2} \times Q \rightarrow E_{1} \times Q$ is $\left(p_{1} \circ \text { proj }\right)^{-1}(\varepsilon)$-homotopic to a homeomorphism, where the size of $\varepsilon$ is determined by the mesh of the partition $\left\{t_{i}\right\}$ of $[0,1]$. This homeomorphism is constructed by piecing together $k+1$ homeomorphisms with disjoint supports, each support containing a cancelling pair.

For the remainder of this case we examine $f_{1} f_{2}: E_{2} \rightarrow E$. The situation is now somewhat improved because the torsion of each $f_{1} f_{2} \mid: p_{2}^{-1}\left(t_{2 i}\right) \rightarrow p^{-1}\left(t_{2 i}\right)$ is 0 by (1) above. The next step is to trade off the torsions

$$
\begin{aligned}
\tau_{2 i} & =f_{1} f_{2} \mid: p_{2}^{-1}\left(\left[t_{2 i}, t_{2 i+2}\right]\right) \rightarrow p^{-1}\left(\left[t_{2 i}, t_{2 i+2}\right]\right), \quad 0 \leqslant i \leqslant k-1, \\
\tau_{2 k} & =f_{1} f_{2} \mid: p_{2}^{-1}\left(\left[t_{2 k}, 1\right]\right) \rightarrow p^{-1}\left(\left[t_{2 k}, 1\right]\right) .
\end{aligned}
$$

Observe that by the Sum Theorem we have $\tau_{0}+\tau_{2}+\cdots+\tau_{2 k}=\tau_{B}$. Now choose an additional subdivision $c_{2 i}<t_{2 i}<d_{2 i}, 1 \leqslant i \leqslant k$, where $c_{2 i}$ and $d_{2 i}$ are close to $t_{2 i}$. Just as $E_{2}$ was formed from $E_{1}$ we now form

$$
E_{3}=E_{2} \cup\left(\bigcup_{i=1}^{k} C_{i}\right) \cup\left(\bigcup_{i=1}^{k} D_{i}\right) \text {. }
$$

It is easy to choose PL sdr's $C_{i} \rightarrow p_{2}^{-1}\left(c_{2 i}\right)$ and $D_{i} \rightarrow p_{2}^{-1}\left(d_{2 i}\right)$ which piece together to give a f.p. $\operatorname{sdr} f_{3}: E_{3} \rightarrow E_{2}$ for which

(1) $\tau\left(f_{3} \mid C_{i}\right)+\left(\tau_{0}+\cdots+\tau_{2 i-2}\right)=0,1 \leqslant i \leqslant k$,

(2) $\tau\left(f_{3} \mid D_{i}\right)-\left(\tau_{0}+\cdots+\tau_{2 i-2}\right)=0,1 \leqslant i \leqslant k$.

If we define $p_{3}=p_{2} f_{3}: E_{3} \rightarrow B$, then $p_{3}$ is a PL approximate fibration. Since we have added torsions to $E_{2}$ in adjacent cancelling pairs we conclude that $f_{3} \times$ id: $E_{3} \times Q$ $\rightarrow E_{2} \times Q$ is $\left(p_{2} \circ \text { proj }\right)^{-1}(\varepsilon)$-homotopic to a homeomorphism, where $\varepsilon$ can be made as small as we want by choosing the differences $d_{2 i}-c_{2 i}$ small. For the remainder of this case we only have to consider $f_{1} f_{2} f_{3}: E_{3} \rightarrow E$. We still have the torsion of each $f_{1} f_{2} f_{3} \mid: p_{3}^{-1}\left(t_{2 i}\right) \rightarrow p^{-1}\left(t_{2 i}\right)$ zero. But, moreover, we now have the torsion of

$$
f_{1} f_{2} f_{3} \mid: p_{3}^{-1}\left(\left[t_{2 i}, t_{2 i+2}\right]\right) \rightarrow p^{-1}\left(\left[t_{2 i}, t_{2 i+2}\right]\right)
$$

zero, and the torsion of

$$
f_{1} f_{2} f_{3} \mid: p_{3}^{-1}\left(\left[t_{2 k}, 1\right]\right) \rightarrow p^{-1}\left(\left[t_{2 k}, 1\right]\right)
$$

is $\tau_{0}+\cdots+\tau_{2 k}$. So what we have done so far is to show how to concentrate $\tau_{B}$ over $\left[t_{2 k}, 1\right]$. It is clear that by applying the same ideas again we can construct a PL approximate fibration $p_{4}: E_{4} \rightarrow B$ and a f.p. PL sdr $f_{4}: E_{4} \rightarrow E_{3}$ so that:

(1) $f_{4} \times$ id: $E_{4} \times Q \rightarrow E_{3} \times Q$ is $(p \circ \text { proj })^{-1}(\varepsilon)$-homotopic to a homeomorphism, where the size of $\varepsilon$ depends on $1-t_{2 k}$;

(2) the torsion of $f_{1} f_{2} f_{3} f_{4} \mid: p_{4}^{-1}\left(t_{2 i}\right) \rightarrow p^{-1}\left(t_{2 i}\right)$ is 0 , for all $i$;

(3) the torsion of $f_{1} f_{2} f_{3} f_{4} \mid: p_{4}^{-1}\left(\left[t_{2 i}, t_{2 i+2}\right]\right) \rightarrow p^{-1}\left(\left[t_{2 i}, t_{2 i+2}\right]\right)$ is 0 , for all $i$;

(4) the torsion of $f_{1} f_{2} f_{3} f_{4} \mid: p_{4}^{-1}\left(\left[t_{2 k}, 1\right]\right) \rightarrow p^{-1}\left(\left[t_{2 k}, 1\right]\right)$ equals the torsion of $f_{1} f_{2} f_{3} f_{4} \mid: p_{4}^{-1}(1) \rightarrow p^{-1}(1)$.

Thus the torsion of $f_{1} f_{2} f_{3} f_{4}: E_{4} \rightarrow E$ is concentrated over *.

We now use the above ideas to finish the proof of the lemma. For arbitrary $B$ we can trade torsions to concentrate $\tau_{B}$ over $*$. By this we mean that there is a PL approximate fibration $p^{\prime}: E^{\prime} \rightarrow B$ and a f.p. PL sdr $f^{\prime}: E^{\prime} \rightarrow E_{1}$ so that:

(1) $f^{\prime} \times$ id: $E^{\prime} \times Q \rightarrow E_{1} \times Q$ is $\left(p_{1} \circ \text { proj }\right)^{-1}(\varepsilon)$-homotopic to a homeomorphism, where $\varepsilon$ is small; 
(2) $\tau\left(\left(p^{\prime}\right)^{-1}(\Delta) \stackrel{f_{1} f^{\prime} \mid}{\rightarrow} p^{-1}(\Delta)\right)$ is 0 , where $\Delta$ is any simplex in $B^{(r)}$ which does not contain $*\left(B^{(r)}=r\right.$ th barycentric subdivision of $B, r$ large $)$;

(3)

$$
\tau\left(\left(p^{\prime}\right)^{-1}(\Delta) \stackrel{f_{1} f^{\prime} \mid}{\rightarrow} p^{-1}(\Delta)\right)=\tau\left(\left(p^{\prime}\right)^{-1}(*) \stackrel{f_{1} f^{\prime} \mid}{\rightarrow} p^{-1}(*)\right)=\tau_{B},
$$

where $\Delta$ is any simplex in $B^{(r)}$ which does contain *.

Since we are given $\tau_{B}=0$ we have $\tau\left(\left(p^{\prime}\right)^{-1}(\Delta) \rightarrow p^{-1}(\Delta)\right)=0$, for all simplices $\Delta$ in $B^{(r)}$. We will use this to show that $f_{1} f^{\prime} \times$ id: $E^{\prime} \times Q \rightarrow E \times Q$ is homotopic to a homeomorphism, where the size of the homotopy in $B$ is determined by the diameters of the simplices in $B^{(r)}$. If $r$ is large enough this homotopy must then be small enough to fulfill our requirements.

By the Classification Theorem of [8] it follows that each

$$
f_{1} f^{\prime} \times \mathrm{id} \mid:\left(p^{\prime}\right)^{-1}(\Delta) \times Q \rightarrow p^{-1}(\Delta) \times Q
$$

is homotopic to a homeomorphism. If it is true that each $\left(p^{\prime}\right)^{-1}(\partial \Delta)$ is a $Z$-set in $\left(p^{\prime}\right)^{-1}(\Delta)$ and each $p^{-1}(\partial \Delta)$ is a $Z$-set in $p^{-1}(\Delta)$, then it is easy to use the Classification Theorem and Z-set unknotting to build a homotopy of $f_{1} f^{\prime} \times$ id to a homeomorphism which is block-preserving, i.e., the restriction of this homotopy to each $\left(p^{\prime}\right)^{-1}(\Delta) \times Q$ has image in $p^{-1}(\Delta) \times Q$. Since the $\Delta$ 's are simplices of $B^{(r)}$ we are done. If the $Z$-set property is not satisfied, then we can construct new PL approximate fibrations $\tilde{p}^{\prime}: \tilde{E}^{\prime} \rightarrow B, \tilde{p}: \tilde{E} \rightarrow B$ and CE maps $\alpha: \tilde{E}^{\prime} \rightarrow E^{\prime}, \beta: \tilde{E} \rightarrow E$ so that

(1) $\alpha$ and $\beta$ are block-preserving,

(2) each $\left(\tilde{p}^{\prime}\right)^{-1}(\partial \Delta)$ is a $Z$-set in $\left(\tilde{p}^{\prime}\right)^{-1}(\Delta)$,

(3) each $(\tilde{p})^{-1}(\partial \Delta)$ is a $Z$-set in $(\tilde{p})^{-1}(\Delta)$.

(To see how to define $\tilde{E}$ for $\operatorname{dim} B=1$ we first let $\tilde{p}^{-1}(v)=p^{-1}(v)$, for all vertices $v$. Then for $\Delta$ a 1-simplex we define

$$
\tilde{p}^{-1}(\Delta)=p^{-1}(\Delta) \cup\left(p^{-1}(\partial \Delta) \times[0,1]\right)
$$

(union along $p^{-1}(\partial \Delta) \equiv p^{-1}(\partial \Delta) \times\{0\}$ ), where $\tilde{p}^{-1}(\Delta)$ is sewn to $\tilde{p}^{-1}(\partial \Delta$ ) along $p^{-1}(\partial \Delta) \times\{1\}$. In general the construction is inductive.) Now $\alpha$ and $\beta$ stabilize to near homeomorphisms [8,p. 105], and the block-preserving property (1) assures us that we have appropriate small control in $B$. So all we need is a homeomorphism $h$ : $\tilde{E}^{\prime} \times Q \rightarrow \tilde{E} \times Q$ for which the following diagram $(p \circ \operatorname{proj})^{-1}(\varepsilon)$-homotopy commutes:

$$
\begin{array}{ccc}
\tilde{E}^{\prime} \times Q & \stackrel{h}{\rightarrow} & \tilde{E} \times Q \\
\alpha \times \mathrm{id} \downarrow & & \downarrow \beta \times \text { id } \\
E^{\prime} \times Q & \stackrel{f_{1} f^{\prime} \times \text { id }}{\rightarrow} & E \times Q
\end{array}
$$

This is easily obtained by first noting that there is a map $H: \tilde{E}^{\prime} \rightarrow \tilde{E}$ which is block-preserving and for which $\beta H$ is block-preserving homotopic to $f_{1} f^{\prime} \alpha$. Then 
$H \mid:\left(\tilde{p}^{\prime}\right)^{-1}(\Delta) \rightarrow(\tilde{p})^{-1}(\Delta)$ has zero torsion, for all simplices $\Delta$ of $B^{(r)}$, and we can now use $Z$-set unknotting to obtain a homotopy of $H \times \mathrm{id}_{Q}$ to a homeomorphism.

We are now ready for our main result. In the following statement $B$ will be a compact connected polyhedron and $p$ will denote projection to $B$.

THEOREM 5.2. If $F$ is a finitely dominated polyhedron, then the condition $\chi(B) \cdot \sigma(F)$ $=0$ is necessary and sufficient that for every $\varepsilon>0$ there exists a compact polyhedron $K$ and $a p^{-1}(\varepsilon)$-equivalence $K \rightarrow B \times F$.

Proof. If such a $K$ exists, then we have $\sigma(B \times F)=0$. By an induction on $\operatorname{dim} B$ it is easy to use the Sum Theorem for the Wall obstruction to prove that $\sigma(B \times F)$ $=i_{*} \chi(B) \cdot \sigma(F)$, where $i: F \rightarrow B \times F$ is defined by $i(x)=(*, x)$. But $i$ has a left inverse, so by the functorality of $\sigma$ we have $\chi(B) \cdot \sigma(F)=0$.

For the other half of the result we assume that $\chi(B) \cdot \sigma(F)=0$. Since $F$ is finitely dominated there is a compact polyhedron $X$ and maps $d: X \rightarrow F, u: F \rightarrow X$ for which $d u: F \rightarrow F$ is homotopic to id ${ }_{F}$. Let $e=u d: X \rightarrow X$ and form the mapping telescope, $S_{e}$, which was introduced in the proof of Lemma 3.1. We also need the mapping torus, $T_{e}=M_{e} / \sim$, where $\sim$ is the equivalence relation on the mapping cylinder $M_{e}$ which is generated by $(x, 0) \sim e(x)$. If exp: $R \rightarrow S^{1}$ is defined by $\exp (x)=e^{\pi i x}$, then it follows from the proof of Theorem 3.1 of [13] that there are homotopy equivalences $H: T_{e} \rightarrow F \times S^{1}$ and $\tilde{H}: S_{e} \rightarrow F \times R$ for which the following diagram commutes:

$$
\begin{array}{ccc}
S_{e} & \stackrel{\tilde{H}}{\rightarrow} & F \times R \\
\downarrow & & \downarrow \text { id } \times \exp \\
T_{e} & \stackrel{H}{\rightarrow} & F \times S^{1}
\end{array}
$$

The map $S_{e} \rightarrow T_{e}$ is the obvious covering map obtained by identifying each $(x, t)$ in $S_{e}$ with the equivalence class of $(x, t)$ in $M_{e} / \sim$.

By reversing the ends of $R$ we similarly obtain the reverse mapping telescope, $S_{e}^{-}$, which is pictured as follows:

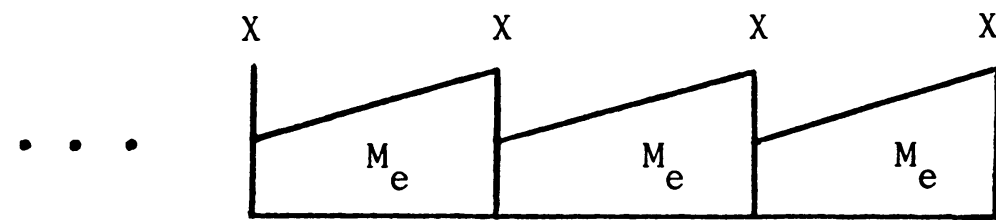

Note that rays of each mapping cylinder go from right to left. Similarly we obtain the reverse mapping torus, $T_{e}^{-}$, and we obtain homotopy equivalences $H_{-}: T_{e}^{-} \rightarrow F \times$ $S^{1}, \tilde{H}_{-}: S_{e}^{-} \rightarrow F \times R$ for which the following diagram commutes:

$$
\begin{array}{ccc}
S_{e}^{-} & \stackrel{\tilde{H}_{-}}{\rightarrow} & F \times R \\
\downarrow & & \downarrow \text { id } \times \exp \\
T_{e}^{-} & \stackrel{H_{-}}{\rightarrow} & F \times S^{1}
\end{array}
$$


In the natural set-wise identification $S_{e} \equiv X \times R$ we observe that each $X \times[n, n+1]$ is a copy of $M_{e}$ with $X \times\{n+1\}$ being the base. In the natural set-wise identification $S_{e}^{-} \equiv X \times R$ we have each $X \times[n, n+1]$ also a copy of $M_{e}$, but $X \times\{n\}$ is now the base. The maps $\tilde{H}$ and $\tilde{H}_{-}$are actually proper homotopy equivalences which preserve the induced orientations. We can choose a homotopy inverse of $H_{-}, H_{-}^{-1}$ : $F \times S^{1} \rightarrow T_{e}^{-}$, and a proper homotopy inverse of $\tilde{H}_{-}, \tilde{H}_{-}^{-1}: F \times R \rightarrow S_{e}^{-}$, so that $\tilde{H}_{-}^{-1}$ covers $H_{-}^{-1}$.

Armed with the above notation we are now ready to make use of Lemma 5.1. If $e$ is chosen to be PL, then $T_{e}$ and $T_{e}^{-}$are compact polyhedra. Thus the projection maps $q: B \times T_{e} \rightarrow B$ and $q_{-}: B \times T_{e}^{-} \rightarrow B$ are PL approximate fibrations. We also have a f.p. homotopy equivalence, id $\times H_{-}^{-1} H: B \times T_{e} \rightarrow B \times T_{e}^{-}$. In order to apply Lemma 5.1 to this map it should, strictly speaking, be a f.p. sdr. By stabilizing $B \times T_{e}$ (i.e. multiplying by a large cell) we could easily replace $H_{-}^{-1} H$ by a sdr, so we will assume that Lemma 5.1 applies to id $\times H_{-}^{-1} H$. To use Lemma 5.1 we need to know that $\tau_{B}=0$, and by the Sum Theorem it follows that $\tau_{B}=\chi(B) \tau\left(H_{-}^{-1} H\right)$. We showed in [5] that the image of $\tau\left(H_{-}^{-1} H\right)$ in $\mathrm{Wh}\left(F \times S^{1}\right)$ under the isomorphism $\left(H_{-}\right)_{*}$ : $\mathrm{Wh}\left(T_{e}^{-}\right) \rightarrow \mathrm{Wh}\left(F \times S^{1}\right)$ is just $\sigma(F)$, where $\tilde{K}_{0}(F)$ is identified with a subgroup of $\mathrm{Wh}\left(F \times S^{1}\right)$ in the usual way [11]. Since $\chi(B) \sigma(F)=0$ we have $\tau_{B}=0$.

Now using Lemma 5.1 there is a homeomorphism $h: B \times T_{e} \times Q \rightarrow B \times T_{e}^{-} \times Q$ which is $\left(q_{-} \circ \text { proj }\right)^{-1}(\varepsilon)$-homotopic to $\mathrm{id}_{B} \times H_{-}^{-1} H \times \mathrm{id}_{Q}$. This homotopy lifts to a proper homotopy $\mathrm{id}_{B} \times \tilde{H}_{-}^{-1} \tilde{H} \times \mathrm{id}_{Q} \simeq \tilde{h}$, where $\tilde{h}: B \times S_{e} \times Q \rightarrow B \times S_{e}^{-} \times Q$ is a homeomorphism. Choose $N$ large enough so that $\tilde{h}^{-1}(B \times X \times[N, \infty) \times Q)$ lies in $B \times X \times(0, \infty) \times Q$. Define

$$
M=(B \times X \times[0, \infty) \times Q) \cap\left(\tilde{h}^{-1}(B \times X \times(-\infty, N] \times Q)\right) .
$$

This is a compact $Q$-manifold. The triangle

$$
\begin{array}{ccc}
B \times S_{e} \times Q & \stackrel{\tilde{h}}{\rightarrow} & B \times S_{e}^{-} \times Q \\
\operatorname{proj} \searrow & & \swarrow \text { proj } \\
& B &
\end{array}
$$

$\varepsilon$-commutes, so using mapping cylinder collapses in $S_{e}$ and $S_{e}^{-}$we observe that the inclusion $M \hookrightarrow B \times S_{e} \times Q$ is a $\pi^{-1}(\varepsilon)$-equivalence, where $\pi=$ proj: $B \times S_{e} \times Q \rightarrow$ $B$. Then the composition

$$
M \hookrightarrow B \times S_{e} \times Q \stackrel{\text { id } \times \tilde{H} \times \text { id }}{\rightarrow} B \times F \times R \times Q \stackrel{\text { proj }}{\rightarrow} B \times F
$$

is a $p^{-1}(\varepsilon)$-equivalence, and since $M$ can be triangulated we are done.

For the proof of Theorem 2 we will need the following version of Theorem 5.2 with dimension estimates.

THEOREM 5.3. For every integer $m$ there exists an integer $n$ so that if $F$ is a finitely dominated polyhedron of dimension $\leqslant n$ and $B$ is a compact connected polyhedron of dimension $\leqslant n$, then the condition $\chi(B) \cdot \sigma(F)=0$ is necessary and sufficient that for every $\varepsilon>0$ there exists a compact polyhedron $K$ of dimension $\leqslant m$ and $a p^{-1}(\varepsilon)$-equivalence $K \rightarrow B \times F$. 
Proof. We can modify the proof of Theorem 5.2, being careful that the dimensions do not grow too large. First we note that since $F$ is finitely dominated and $\operatorname{dim} F \leqslant n$, we can choose the dimension of the dominating compact polyhedron $X$ to be $\leqslant n$. This just uses the fact that any map of $F$ into a polyhedron can be homotoped into the $n$-skeleton. The next change in the proof of Theorem 5.2 comes when the homeomorphism $h$ is constructed. We will replace this with a compact polyhedron $Z$ and CE-PL maps $\alpha: Z \rightarrow B \times T_{e}, \beta: Z \rightarrow B \times T_{e}^{-}$such that $\beta$ is $\left(q_{-}\right)^{-1}(\varepsilon)$-homotopic to $\left(\operatorname{id}_{B} \times H_{-}^{-1} H\right) \alpha$. Also we want $\operatorname{dim} Z$ to be bounded in terms of $n$. Once we have done this we lift $\alpha, \beta$ to $\tilde{\alpha}: \tilde{Z} \rightarrow B \times S_{e}, \quad \tilde{\beta}: \tilde{Z} \rightarrow B \times S_{e}^{-}$, and our desired compact polyhedron $K$ is

$$
K=\tilde{\alpha}^{-1}(B \times X \times[0, \infty)) \cap \tilde{\beta}^{-1}(B \times X \times(-\infty, N]),
$$

for $N$ a large integer.

To construct $Z$ and the CE-PL maps $\alpha, \beta$ we need to improve Lemma 5.1. Using the notation of Lemma 5.1 suppose that $\operatorname{dim} E, \operatorname{dim} E_{1}$ and $\operatorname{dim} B$ are all $\leqslant n$. We want to prove that if $\tau_{B}=0$, then for every $\varepsilon>0$ there exists a compact polyhedron $Z, \operatorname{dim} Z \leqslant m$, and CE-PL maps $\alpha: Z \rightarrow E_{1}, \beta: Z \rightarrow E$ so that $\beta$ is $p^{-1}(\varepsilon)$-homotopic to $f_{1} \alpha$. Of course $m$ should depend only on $n$. Recall that the strategy used in Lemma 5.1 was to use a trick of trading adjacent cancelling torsions so that we were eventually reduced to the case in which the torsion of $f_{1} \mid: p_{1}^{-1}(\Delta) \rightarrow p^{-1}(\Delta)$ is zero, for all simplices in $B^{(r)}$. We first explain how to redo this trick in the context of our desired improvement of Lemma 5.1.

Suppose that $a_{1}, a_{2} \in B$ are close together and we have defined $E_{2}=E_{1} \cup A_{1} \cup$ $A_{2}$, where there are sdr's $A_{i} \rightarrow p_{1}^{-1}\left(a_{i}\right)$ which piece together to yield a f.p. PL sdr $f_{2}$ : $E_{2} \rightarrow E_{1}$. This gives us a PL approximate fibration $p_{2}=p_{1} f_{2}: E_{2} \rightarrow B$. Also we assume that the torsions of $f_{2} \mid: p_{2}^{-1}\left(a_{1}\right) \rightarrow p_{1}^{-1}\left(a_{1}\right)$ and $f_{2} \mid: p_{2}^{-1}\left(a_{2}\right) \rightarrow p_{1}^{-1}\left(a_{2}\right)$ add up to zero in $\mathrm{Wh}\left(p^{-1}(*)\right)$. Thus they add up to zero over a small polyhedron $P$ in $B$ which contains a PL arc from $a_{1}$ to $a_{2}$. This means that $f_{2} \mid: p_{2}^{-1}(P) \rightarrow p_{1}^{-1}(P)$ has zero torsion, and so we can find a compact polyhedron $W$ and CE-PL retractions $u$ : $W \rightarrow p_{2}^{-1}(P), v: W \rightarrow p_{1}^{-1}(P)$. Define $E_{3}=E_{2} \cup W$, sewn together along $p_{2}^{-1}(P)$, and define $f_{3}: E_{3} \rightarrow E_{2}$ by $f_{3} \mid W=u$ and $\mathrm{f}_{3}=$ id otherwise. Now $p_{3}=p_{2} f_{3}: E_{3} \rightarrow B$ is still an approximate fibration and the torsion of $f_{1} f_{2} f_{3} \mid: p_{3}^{-1}\left(a_{i}\right) \rightarrow p^{-1}\left(a_{i}\right)$ equals the torsion of $f_{1} f_{2} \mid: p_{2}^{-1}\left(a_{i}\right) \rightarrow p^{-1}\left(a_{i}\right)$, for $i=1,2$. Also the torsion of $f_{1} f_{2} f_{3} \mid:$ $p_{3}^{-1}(b) \rightarrow p^{-1}(b)$ equals the torsion of $f_{1} \mid: p_{1}^{-1}(b) \rightarrow p^{-1}(b)$, for all $b \neq a_{1}, a_{2}$ in $B$. So in the context of trading adjacent torsions the space $E_{3}$ is as good as the space $E_{2}$. However we now have the advantage that $v$ extends to a CE-PL map $\tilde{v}: E_{3} \rightarrow E_{1}$ for which $\tilde{v}$ is homotopic to $f_{2} f_{3}$ via a homotopy whose image in $B$ is small provided that the diameter of $P$ is small. So if the lemma is true for $p_{3}: E_{3} \rightarrow B$ it is also true for $p_{1}: E_{1} \rightarrow B$.

The above comments tell us that we can now use the torsion trading trick to reduce ourselves to the special case in which the torsion of each $f_{1} \mid: p_{1}^{-1}(\Delta) \rightarrow p^{-1}(\Delta)$ is zero. We will inductively build our desired $Z$ and CE-PL retractions $\alpha: Z \rightarrow E_{1}, \beta$ : $Z \rightarrow E$. For each $i$ let $B_{i}$ denote the $i$-skeleton of $B$. Note that for each vertex $v$ of $B_{0}$, the inclusion $p^{-1}(v) \hookrightarrow p_{1}^{-1}(v)$ has torsion 0 . Thus by expanding each $p_{1}^{-1}(v)$ we can 
form a compact polyhedron $Z_{0}$ and CE-PL retractions $\alpha_{0}: Z_{0} \rightarrow E_{1}, \beta_{0}$ : $\left(p_{1} \alpha_{0}\right)^{-1}\left(B_{0}\right) \cup E \rightarrow E$ so that $\beta_{0}\left(\left(p_{1} \alpha_{0}\right)^{-1}(v)\right)=p^{-1}(v)$, for each $v$ in $B_{0}$. This completes the first step of the induction. Next note that for each 1-simplex $\Delta$ of $B_{1}$, the inclusion $\left(p \beta_{0}\right)^{-1}(\Delta) \hookrightarrow\left(p_{1} \alpha_{0}\right)^{-1}(\Delta)$ has torsion 0 . Thus by expanding each $\left(p_{1} \alpha_{0}\right)^{-1}(\Delta)$ we can form a compact polyhedron $Z_{1}$ and CE-PL retractions

$$
\alpha_{1}: Z_{1} \rightarrow Z_{0}, \quad \beta_{1}:\left(p_{1} \alpha_{0} \alpha_{1}\right)^{-1}\left(B_{1}\right) \cup E \rightarrow\left(p_{1} \alpha_{0}\right)^{-1}\left(B_{0}\right) \cup E
$$

so that $\beta_{1}$ takes each $\left(p_{1} \alpha_{0} \alpha_{1}\right)^{-1}(\Delta)$ to $\left(p_{1} \alpha_{0}\right)^{-1}(\partial \Delta) \cup p^{-1}(\Delta)$. By composing these maps with $\alpha_{0}$ and $\beta_{0}$ we retrieve CE-PL retractions

$$
\alpha_{0} \alpha_{1}: Z_{1} \rightarrow E_{1}, \quad \beta_{0} \beta_{1}:\left(p_{1} \alpha_{0} \alpha_{1}\right)^{-1}\left(B_{1}\right) \cup E \rightarrow E .
$$

This completes the second step of the induction. It should now be clear how this process can be continued to eventually find our desired $Z$ and CE-PL retractions $\alpha, \beta$.

6. Proof of Theorem 2 (infinite-dimensional case). We start by treating the case in which $B$ is a compact connected polyhedron. Choose a finitely dominated polyhedron $F$, with Wall finiteness obstruction $\sigma(F) \in \tilde{K}_{0}(F)$, for which $\chi(B) \cdot \sigma(F)=$ 0 . By Theorem 5.2 there is a compact $Q$-manifold $M$ and a $\pi^{-1}(\varepsilon)$-equivalence $f$ : $M \rightarrow B \times F$, where $\pi=$ proj: $B \times F \rightarrow B$ and $\varepsilon$ is small. Then $\pi f: M \rightarrow B$ is an $\varepsilon^{\prime}$-fibration, for $\varepsilon^{\prime}$ small, and so by Theorem 1 of [6] $\pi f$ is close to an appropriate fibration $p: M \rightarrow B$. Homotopic maps have homotopy fibers which are homotopy equivalent, so $p$ has fiber $F$.

For the general case in which $B$ is a compact connected ANR we form $B \times Q$ and write $B \times Q=B^{\prime} \times Q$, where $B^{\prime}$ is a compact polyhedron. Let $F$ be a finitely dominated polyhedron for which $\chi(B) \cdot \sigma(F)=\chi\left(B^{\prime}\right) \cdot \sigma(F)=0$, and use the above case to get an approximate fibration $p^{\prime}: M \rightarrow B^{\prime}$ with fiber $F$. Then $p^{\prime} \times$ id: $M \times Q \rightarrow B^{\prime} \times Q=B$ is also an approximate fibration with fiber $F$.

7. Proof of Theorem 2 (finite-dimensional case). In the language of [19] our first step is to choose a PL manifold $W^{w}, w \geqslant 5$, which has a single tame end $\infty$ and an end invariant $\sigma(\infty) \in \tilde{K}_{0} \pi_{1}(\infty)$ which is an obstruction to putting a boundary on $W$. Recall that such a $W$ has the property that $\pi_{1}$ is stable at $\infty$ and for each cofinite subcomplex $K$ of $W, K$ is finitely dominated and its finiteness obstruction is just $\sigma(\infty)$ (up to sign). By [19, Chapter VIII] all such elements of a given $\tilde{K}_{0} \pi_{1}(\infty)$ can be realized in this manner, and if we have $\pi_{1}(\infty)=Z_{257}$, then we can choose $\sigma(\infty)$ so that $\sigma(\infty)+(-1)^{w-1} \overline{\sigma(\infty)} \neq 0$, where ${ }^{-}: \tilde{K}_{0} \pi_{1}(\infty) \rightarrow \tilde{K}_{0} \pi_{1}(\infty)$ is the duality involution [19, p. 118].

For our next step we will need a construction from [14] which provides us with an open neighborhood $U \subset W$ of $\infty$ for which there is an approximate fibration $q$ : $U \rightarrow R$ such that $q^{-1}([0, \infty))$ is a closed neighborhood of $\infty$. Here is a brief description of how $U$ arises: Using engulfing it is possible to perform the twistgluing operation of [20] to wrap up the ends of a neighborhood $V$ of $\infty$ to obtain a compact manifold $N^{w+1}$ and a $\delta$-fibration $u: N \rightarrow S^{1}$, for $\delta$ small. Applying 
Theorem 1 of [7] we see that $u$ is close to an approximate fibration $v: N \rightarrow S^{1}$. The pull-back

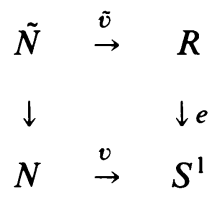

provides us with an approximate fibration $\tilde{v}: \tilde{N} \rightarrow R$ for which $\tilde{v}^{-1}((-1,1))$ is identified with a "sizable" open subset of $V$. Then engulfing enables us to stretch out this open set to obtain an identification of all $\tilde{N}$ with an open neighborhood $U$ of $\infty$ which approximately fibers over $R$ as desired. Note that $U$ is finitely dominated and it has two tame ends, $\infty_{-}$and $\infty_{+}=\infty$. Write $U=K_{-} \cup K_{+}$, where $K_{-}$and $K_{+}$are closed subcomplexes for which $K_{-} \subset q^{-1}((-\infty, 1])$ and $K_{+} \subset q^{-1}([-1, \infty))$. By the Sum Theorem we have $\sigma(U)=\sigma\left(K_{-}\right)+\sigma\left(K_{+}\right)$, thus $\sigma(U)=\sigma\left(\infty_{-}\right)+\sigma(\infty)$ (up to sign). By the duality formula of $\left[19\right.$, p. 119] we have $\sigma\left(\infty_{-}\right)=(-1)^{w-1} \overline{\sigma(\infty)}$. Since we have chosen $\sigma(\infty)$ so that $\sigma(\infty)+(-1)^{w-1} \overline{\sigma(\infty)} \neq 0$ we conclude that $U$ is not homotopy equivalent to a finite complex.

Now consider the product $B^{n} \times W$, there $B^{n}$ is our given base manifold of Theorem 2 which has Euler characteristic zero. We are going to carve out of $B \times W$ an approximate fibration $p: M^{k} \rightarrow B$, where $k=n+w-1$ and the homotopy fiber of $p$ is $U$. To do this we apply the Approximate End Theorem of Quinn [18] to the map $\pi=$ proj: $B \times W \rightarrow B$. In what follows it will be helpful for the reader to be acquainted with $\$ 7$ of [18] in which the Approximate End Theorem is established. For the time being we will specifically assume that the obstruction which occurs at the bottom of p. 319 of [18] is zero, and show how this yields our desired approximate fibration $p: M \rightarrow B$. Since this obstruction is zero we can carve up $B \times W$ into $h$-cobordisms where the projection of the homotopies to $B$ are small. More precisely we can find compact clean PL submanifolds $N_{1}, N_{2}$ of $B \times U$ so that:

(1) $\partial N_{1}=\partial_{-} N_{1} \cup \partial_{+} N_{1}$ and $\partial N_{2}=\partial_{-} N_{2} \cup \partial_{+} N_{2}$;

(2) $N_{1} \cap N_{2}=\partial_{+} N_{1}=\partial_{-} N_{2}$;

(3) $\partial_{-} N_{1} \subset B \times q^{-1}((-3,-2))$, $\partial_{+} N_{1} \subset B \times q^{-1}((-1,1)), \partial_{+} N_{2} \subset B \times q^{-1}((2,3))$;

(4) $B \times q^{-1}((-2,-1)) \subset N_{1} \subset B \times q^{-1}((-3,1))$;

(5) $B \times q^{-1}((1,2)) \subset N_{2} \subset B \times q^{-1}((-1,3))$;

(6) there are $\pi^{-1}(\delta)$-sdr's of $N_{1}$ onto $\partial_{-} N_{1}$ and onto $\partial_{+} N_{1}$;

(7) there are $\pi^{-1}(\delta)$-sdr's of $N_{2}$ onto $\partial_{-} N_{2}$ and onto $\partial_{+} N_{2}$.It then follows that there is a $\pi^{-1}(\delta)$-sdr of $B \times U$ onto $\partial_{+} N_{1}$. Since $\pi: B \times U \rightarrow B$ is a Hurewicz fibration we conclude that $\pi \mid: \partial_{+} N_{1} \rightarrow B$ is a $\delta$-fibration whose homotopy fiber is also $U$. By Theorem 1 of [7], $\pi \mid \partial_{+} N_{1}$ is close to an approximate fibration $p: \partial_{+} N_{1} \rightarrow B$ with homotopy fiber $U$. This completes the proof of Theorem 2 in case the obstruction vanishes. We now show that this obstruction vanishes so that we can construct our desired "controlled" $h$-cobordisms.

The first step is to construct a clean neighborhood $N \subset B \times W$ of $\infty$ for which $(N, \partial N)$ is $(n+w-3)$-connected. In the uncontrolled case we just use Siebenmann's thesis [19], but we want to do this with control in $B$ so we use $\S 7$ of [18] to construct 
$N$ so that $(N, \partial N)$ is $(\delta, n+w-3)$-connected for some small $\delta$ (i.e. all the homotopies have diameters $<\delta$ in the $B$-coordinate). We want to carve out of this a clean neighborhood $N_{1} \subset N$ of $\infty$ for which $\left(N_{1}, \partial N_{1}\right)$ is $(\varepsilon, n+w-2)$-connected for some small $\varepsilon$. Once this is done we can proceed as in [18] to finish the argument. We first choose another $N^{\prime} \subset N$ which is much closer to $\infty$ so that $\left(N^{\prime}, \partial N^{\prime}\right)$ is also $(\delta, n+w-3)$-connected, and then perform a small deformation of the handlebody structure so that

$$
N-\left(N^{\prime}\right)^{\circ}=\partial N \cup\{(n+w-3) \text {-handles }\} \cup\{(n+w-2) \text {-handles }\}
$$

and so that each handle is small. ("Small" generally refers to measurements made in the $B$-coordinate.) Let $A$ be the union of $\partial N$ and the given $(n+w-3)$-handles, and consider the kernel of the boundary map of $Z \pi_{1}(\infty)$-modules,

$$
\text { ว: } \pi_{n+w-2}\left(N-\left(N^{\prime}\right)^{\circ}, A\right) \rightarrow \pi_{n+w-3}(A, \partial N) .
$$

Recall from [18, p. 319] that $\pi_{n+w-2}\left(N-\left(N^{\prime}\right)^{\circ}, A\right)$ may be regarded as a geometric $Z \pi_{1}(\infty)$-module $G$ and there is a small projection $u: G \rightarrow G$ for which $\operatorname{Im} u=\operatorname{Ker} \partial$. Precisely what is needed is the following: There is another geometric $Z \pi_{1}(\infty)$-module $G^{\prime}$ and a small deformation $E: G \oplus G^{\prime} \rightarrow G \oplus G^{\prime}$ so that $E\left(\operatorname{Ker} \partial \oplus G_{1}^{\prime}\right)$ is freely generated by a subset of the preferred bases of $G$ and $G^{\prime}$, where $G_{1}^{\prime} \subset G^{\prime}$ is freely generated by a subset of the preferred basis of $G^{\prime}$. This condition can be met by merely assuming that Ker $\partial$ is stably free with control in $B$, i.e., there are geometric modules $G_{1}, G_{2}$ and a small isomorphism $G_{1} \cong \operatorname{Ker} \partial \oplus G_{2}$. The deformation $E$ comes from Lemma 5.4 of [19] and it is small provided that the data which is plugged into the proof in [19] is small.

Recall in the uncontrolled case of [19] it is shown that if $N$ has the homotopy type of a finite complex, then $\operatorname{Ker} \partial$ is stably free, i.e., there are geometric modules $G_{1}, G_{2}$ and an isomorphism $G_{1} \cong \operatorname{Ker} \partial \oplus G_{2}$. We claim that the same proof gives us a small isomorphism (and therefore Ker $\partial$ is stably free with control) provided that we plug in the additional data that there is a compact polyhedron $K$ and a $\pi^{-1}(\delta)$-equivalence $K \simeq N$, for some small $\delta$. The dimension of $K$ must be bounded in terms of $n+w$, for when one examines the uncontrolled proof of [19] he finds that layers of cells are attached to $N$ to build its dimension up to $\operatorname{dim} K$, and each time a new layer is attached some control is lost in $B$. So there only remains the problem of obtaining $K$. If we choose a closed polyhedral neighborhood $P \subset W$ of $\infty$ for which $B \times P \subset N$, then Theorem 5.3 tells us that there exists a compact polyhedron $K^{\prime}$ and a $\pi^{-1}(\delta)$-equivalence $K^{\prime} \simeq B \times P$. Since $N-(B \times P)^{\circ}$ is a compact polyhedron, then our desired $K$ is obtained from $K^{\prime}$ simply by attaching a finite complex.

\section{REFERENCES}

1. D. R. Anderson and W. C. Hsiang, The functors $K_{-i}$ and pseudo-isotopies of polyhedra, Ann. of Math. (2) 105 (1977), 201-223.

2. H. Bass, Algebraic K-theory, Benjamin, New York, 1968.

3. D. Carter, private communication, (an example), January 1980.

4. T. A. Chapman, Homotopy conditions which detect simple homotopy equivalences, Pacific J. Math. 80 (1979), 13-46. 
5. Whitehead torsion and the Wall finiteness obstruction, Canad. J. Math. (to appear).

6. Approximation results in Hilbert cube manifolds, Trans. Amer. Math. Soc. 262 (1980), $303-334$.

7. __ Approximation results in finite-dimensional manifolds (preprint).

8. L_ Lectures on Hilbert cube manifolds, CBMS Regional Conf. Ser. in Math., no. 28, Amer. Math. Soc., Providence, R. I., 1976.

9. M. Cohen, $A$ course in simple-homotopy theory, Springer-Verlag, Berlin and New York, 1970.

10. D. Coram and P. Duvall, Approximate fibrations, Rocky Mountain J. Math. 7 (1977), 275-288.

11. F. T. Farrell and W. C. Hsiang, $A$ formula for $K_{1}\left(R_{\alpha}[T]\right)$, Proc. Sympos. Pure Math., vol. 17, Amer. Math. Soc., Providence, R. I., 1970.

12. S. Ferry, Approximate fibrations with nonfinite fibers, Proc. Amer. Math. Soc. 64 (1977), 335-345.

13. The homeomorphism group of a compact Hilbert cube manifold is an ANR, Ann. of Math. (2) 106 (1977), 101-119.

14. A Approximate fibrations over $S^{1}$ (in preparation).

15. R. Geoghegan, Open problems in infinite-dimensional topology (preprint).

16. J. F. P. Hudson, Piecewise linear topology, Benjamin, New York, 1969.

17. L. S. Husch, Approximating approximate fibrations by fibrations. II (preprint).

18. F. Quinn, Ends of maps. I, Ann. of Math (2) 110 (1979), 275-331.

19. L. C. Siebenmann, The obstruction to finding a boundary for an open manifold of dimension greater than five, Thesis, Princeton University, 1965.

20. __ A total Whitehead torsion obstruction, Comment. Math. Helv. 45 (1970), 1-48.

DePartment of Mathematics, University of Kentucky, LeXington, Kentucky 40506 